\title{
Management of Abscess in Right Para Lumber Fossa in a Cow - A Case Report
}

\section{Zahid Bashir Khanday*}

Veterinary Assistant Surgeon, Animal Husbandry Kashmir, India

*Corresponding Author: Zahid Bashir Khanday, Veterinary Assistant Surgeon, Animal Husbandry Kashmir, India.
Received: July 03, 2021

Published: August 26, 2021

(C) All rights are reserved by Zahid Bashir

Khanday.

\begin{abstract}
An abscess is an accumulation of pus that hollows out a cavity in the tissues by destroying and expanding them and surrounded by fibrous tissue. Diagnosis of a skin abscess is usually made based on what it looks like and is confirmed by cutting it open. Ultrasound imaging may be useful in cases in which the diagnosis is not clear. Successful management of the abscess was presented in female cattle of five years of age. The abscess swelling was unusually located subcutaneously in right Para lumber fossa, behind the last rib. Clinical examination revealed the mass was warm, hard, and painful in the initial stages, and become fluctuating, gas filled and soft on maturation. The animal was successfully treated by regular surgical drainage of pus along with a daily antiseptic dressing of tincture-iodine, parenteral administration of antibiotics (streptomycin and penicillin, for 5 days), and oxytetracycline wound spray. The animal was treated successfully and it recovered after 1 month post-intervention without any other complications.
\end{abstract}

Keywords: Abscess; Antibiotics; Cattle; Surgical Drainage

\section{Introduction}

\section{Occurrence}

An abscess is an accumulation of pus surrounded by fibrous tissue. They may occur anywhere in the body where pyogenic (pus forming) bacteria can establish and multiply. Over time, pus is mostly replaced by fibrous tissue. Abscesses of the lymph nodes in the head and neck region, and of the skin, are commonly seen as a consequence of abrasions or penetrations of the oropharynx or skin from grazing prickly plants [1]. Golf ball to tennis ball size abscesses on the cheeks and jaw are probably due to skin penetration from thorns or other sharp objects during feeding or rubbing. Subcutaneous abscesses may occur at vaccination sites when vaccination is performed under sub-optimal conditions (wet, dirty or blunt needles). However, it's more difficult to identify an abscess inside the body.

\section{Signs and symptoms}

The symptoms of abscesses include redness, tenderness, pain in the affected area, a high temperature (warmth), and swelling (if abscesses are near the skin layer) or constitutional symptoms (if abscesses are deep).

\section{Diagnosis}

The swelling may feel fluid-filled when pressed. A superficial abscess should be differentiated from other swellings by an exploratory puncture which reveals synovial fluid in bursitis; serous fluid in cysts; blood in hematoma; nothing or intestinal content in the hernia. In most cases, it is recommended to double-check that the swelling is fluid-filled, and not an indurated fibrous lump, hernia, or hematoma before incising. 


\section{Treatment}

Most skin abscesses are harmless and may go away without treatment. However there are cases in which an abscess can lead to serious, potentially life-threatening complications if left untreated. Sometimes, skin abscesses are more difficult to treat and may require laceration or drainage [4]. Most subcutaneous abscesses are the result of traumatic skin penetration with resulting infection. When bacteria enter the body, the white blood cells attack the bacteria and some nearby tissue dies, creating a hole which then fills with pus to form an abscess [2]. The pus contains a mixture of dead tissue, white blood cells, and bacteria. The abscess may get larger and more painful as the infection continues and more pus is produced. Abscesses can be treated in several different ways, depending on the type of abscess and how large it is. The main treatment options include antibiotics, a drainage procedure, and surgery. Proper physical and chemical restraining of the animal (crush, and sedation) may be vital before starting manipulation. Besides, aseptic procedures should be followed during the procedure to reduce infection and increase the prognosis of the condition. A small skin abscess may drain naturally, or simply shrink, dry up and disappear without any treatment. However, large superficial abscesses may need to be treated with antibiotics to clear the infection, and the pus may need to be drained through an incision. This will usually be done by creating a ventral drainage hole and thoroughly flush with clean water under moderate pressure from a hose or syringe using a gloved finger to gently aid the removal of pus [3]. The present case report discusses the successful management of superficial abscess in cattle, which was located in right Para-lumbar fossa, behind the last rib. The said abscess was around 4.5 inches in diameter.

\section{Case History}

Clinical examination and preoperative animal preparation

A case of a 5 year old cow was presented in field conditions with a swelling in right Para lumbar fossa, behind the last rib. History revealed that the cow was from a herd, were other animals of the herd may have gored the said animal. The clinical examination revealed a swelled mass, which was warm, hard, and painful in the initial stages, and become fluctuating and soft for maturation whereas their history revealed the gradual reduction of feed intake and water consumption. All physiological parameters were normal in the animal. To differentiate it from other types of lesions caus- ing swelling like a tumor, hematoma, hernia, etc., aspiration of the content from the swollen mass was performed using an 18-gauge sterile needle that revealed that the swelling was filled with large amounts of gas, followed by a creamy discharge that helped in the diagnosis of the abscess. Finally, surgical evacuation of the pus was conducted to relieve the cattle from this condition. Chemically, the animals were first sedated with xylazine with a dose of $0.2 \mathrm{mg} /$ $\mathrm{kg}$ intramuscularly. Initially the abscess was drained, keeping the animal in standing position. However it was found that the abscess was large and deep seated. For further drainage the animal was then physically restrained with rope, assisted by personnel to adequately restrain it in the lateral position.

\section{Surgical correction}

Following proper physical and chemical restraining and aseptic preparation of the surgical site, surgical evacuation of the pus was performed after proper restraining in lateral recumbency. The hair surrounding the swelling was aseptically prepared by clipping, shaving, scrubbing, washing with water, and Chlorhexidine $4 \%$ solution. An incision with a disposable sterile scalpel was made around the tip of the abscess. A large quantity of pus was drained and the cavity filled with dilute $(0.5 \%)$ tincture iodine. The cavity was then filled with packed with gauze soaked in tincture iodine $(5 \%)$ with a seton protruding out of the incision to allow pus drainage.

\section{Post-operative follow-up and result}

Post-surgery, small skin bleeding was noticed but it is normal and helps to heal the wound unless continued for a long period. The wound was drained and dressed with tincture iodine for five days post-operation followed alternatively for next 3 weeks. Besides, the animal was kept on antibiotic combination of streptomycin (@2.5 g), procaine penicillin (15,00,000 IU) and penicillin G $(5,00,000$ IU) I.M, which was administered for five consecutive days. Meloxicam at a dose rate of $0.5 \mathrm{mg} / \mathrm{kg}$, IM was given twice daily for three successive days for pain management. Moreover, oxytetracycline wound spray was used for five successive days. The postoperative evaluations showed good healing progress of the surgical wound. The whole wound successfully recovered after 1 month without any other complications and no signs of recurrence were seen during this period. 


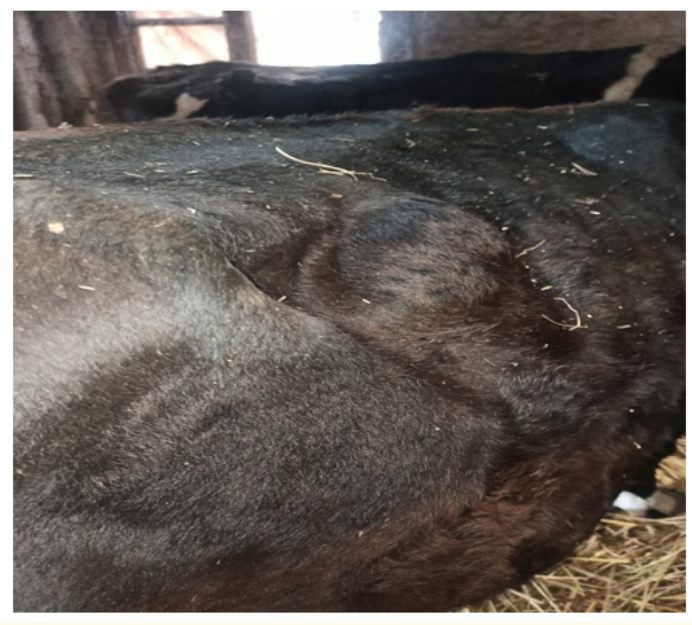

Figure 1: Swelling in Right Paralumbar fossa.

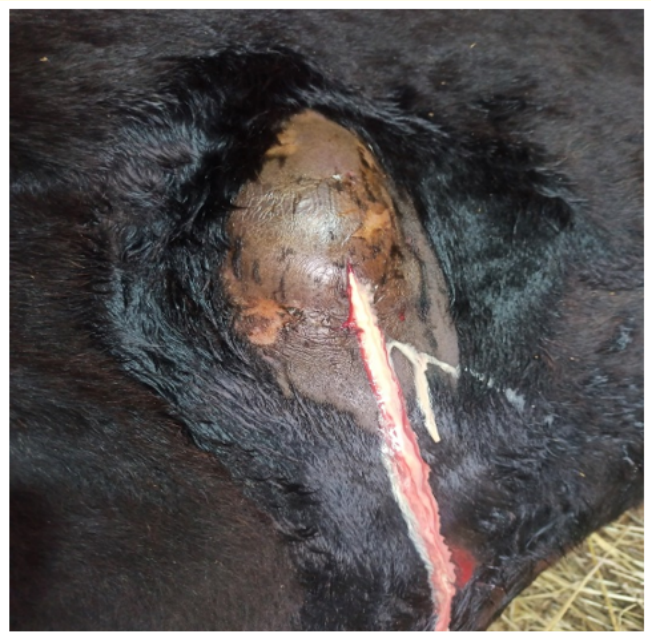

Figure 2: Drainage of Pus.

\section{Conclusion}

An abscess is a circumscribed inflammatory lesion, which consists of purulent exudates. Causes of abscesses formation are variable and include a breach on the surface of the skin or mucous membrane and entrance of pyogenic microorganisms through it, infected foreign bodies migrating from the lumen of the digestive tract, the non-sterilized needle used for intramuscular injection,

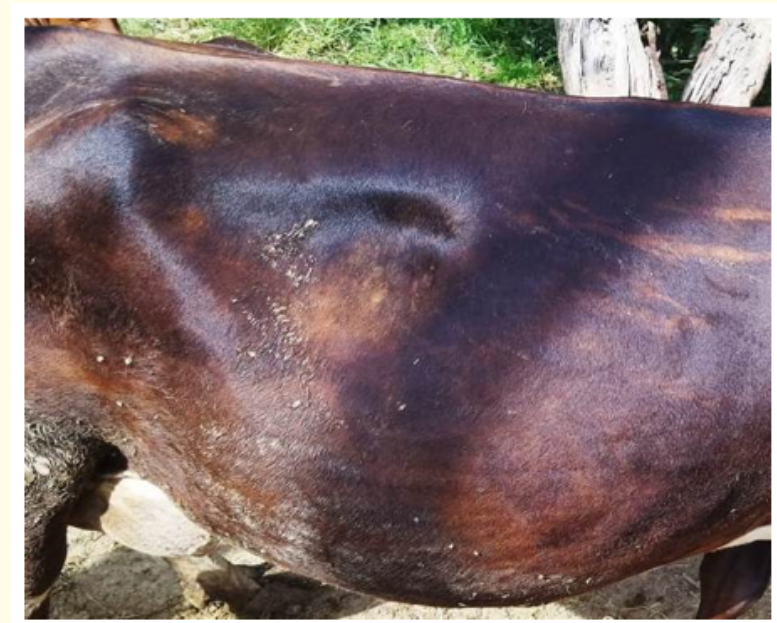

Figure 3: Post Recovery.

punctured or penetrating wounds. Based upon this study, it could be concluded that at field level, successful management of subcutaneous abscess in cattle can be achieved by proper/regular drainage of the pus followed by antiseptic gauze packing to allow drainage of pus so that the parental administration of antibiotics is effective.

\section{Bibliography}

1. Al-Harbi KB. "Prevalence and etiology of abscess disease of sheep and goats at Qassim region, Saudi Arabia”. Veterinary World 4.11 (2011): 495.

2. Blood DC., et al. "Diseases of the alimentary tract, Special examination”. In: Veterinary Medicine, 7th edn. Bailliere and Tindal, London (2000): 160-167.

3. Hassan N., et al. "Management of large subcutaneous abscess in a dairy cow". Journal of Pharmacognosy and Phytochemistry 8.1 (2019): 1652-1653.

4. Kofler J., et al. "Treatment of infected wounds and abscesses in bovine limbs with Ligasano-polyurethane-soft foam dressing material". Berliner und Munchener tierarztliche Wochenschrift 117.9-10 (2004): 428-438.

\section{Volume 3 Issue 10 October 2021}

(C) All rights are reserved by Zahid Bashir Khanday. 